\title{
Estudio mediante EIS de capas lantánidas de conversión desarrolladas mediante activación térmica sobre aleaciones de Al-Mg
}

\author{
M. Bethencourt", F.J. Botana*, M.J. Cano*, M. Montero* y M. Marcos** \\ Resumen En la actualidad, se encuentran en desarrollo una gran variedad de tratamientos \\ anticorrosivos que pueden emplearse como alternativas a los basados en $\mathrm{Cr}(\mathrm{VI})$. Algunos \\ conllevan el empleo de lantánidos. En este trabajo, se lleva a cabo un estudio de las \\ características morfológicas y anticorrosivas de capas desarrolladas sobre la aleación \\ AA5083 a partir de disoluciones de Ce(III), mediante tratamientos de inmersión a 323K \\ en baños de sal de cerio. Estudios SEM/EDS sobre la muestras tratadas han puesto de \\ manifiesto la existencia de una capa heterogénea formada por una película de \\ óxido/hidróxido de aluminio sobre la matriz y una serie de islas dispersas de cerio, sobre los \\ intermetálicos catódicos. Para evaluar el grado de protección de las capas de conversión \\ desarrolladas, así como para caracterizar la contribución individual de la respuesta \\ electroquímica del sistema en $\mathrm{NaCl}$, se han llevado a cabo ensayos de EIS.
}

Palabras clave Aleación AA5083. Capas de conversión. Cerio. Ensayo EIS.

\section{EIS study of lanthanide conversion coatings developed by thermal activation on Al-Mg alloys}

\begin{abstract}
Nowadays, a lot of anticorrosive treatments that can be used as alternative processes to $\mathrm{Cr}(\mathrm{IV})$-based treatments are developing. Some of them involve the use of lanthanide compounds. The present work show a morphologic and anticorrosive study of conversion coatings developed on samples of AA5083 alloy from Ce (III) solutions, by treatments of immersion to $323 \mathrm{~K}$ in baths of cerium salts.

SEM/EDS analysis of treated sampled shows the existence of an heterogeneous coating composed of an aluminium hydroxide/oxide on the metallic matrix and $\mathrm{Ce}$ island over cathodic intermetallics. Protected with conversion coatings, AA5083 samples were immersed in $\mathrm{NaCl}$ and EIS tests were registered both to evaluate the protection degree of the developed coatings and to characterize the individual contribution of the electrochemical response of the system.
\end{abstract}

Keywords $\quad$ AA5083 alloy. Conversión coatings. Cerium. EIS test.

\section{INTRODUCCIÓN}

El análisis microestructural de la aleación AA5083 (Al-Mg) mostró la existencia de tres tipos de compuestos intermetálicos, identificados como $\mathrm{Al}(\mathrm{Mn}, \mathrm{Fe}, \mathrm{Cr}), \mathrm{Al}(\mathrm{Si}, \mathrm{Mg})$ y una fase $\beta$ de $\mathrm{Al}-\mathrm{Mg}^{[1]}$. El comportamiento frente a la corrosión de esta aleación en disoluciones aireadas de $\mathrm{NaCl}$ viene condicionado por la presencia y distribución superficial del primero de esos intermetálicos ${ }^{[1-8]}$. Así, cuando una muestra de la aleación AA5083 se expone a una disolución de $\mathrm{NaCl}$, el principal proce- so de corrosión que tiene lugar en la superficie es la corrosión alcalina localizada (LAC) en el entorno de los precipitados de $\mathrm{Al}(\mathrm{Mn}, \mathrm{Fe}, \mathrm{Cr})$, que actúan como cátodos permanentes, sin que el resto de los precipitados se vean afectados ${ }^{[1}$ y 2$]$. Simultáneamente al proceso LAC, tiene lugar un segundo proceso consistente en la formación de una capa protectora de óxido sobre la matriz metálica cuyo espesor crece con el tiempo de exposición ${ }^{[1]}$.

Por esta razón, el diseño de una sistema de protección efectivo debe contemplar el empleo de inhibidores catódicos. En trabajos anteriores se ha

(*) Departamento de Ciencia de los Materiales e Ingeniería Metalúrgica y Química Inorgánica. Universidad de Cádiz. Facultad de Ciencias del Mar y Ambientales. Avda. República Saharaui s/n, 11510 Puerto Real, Cádiz, España.

(**) Dpto. de Ingeniería Mecánica y Diseño Industrial, Escuela Superior de Ingeniería. C) Chile, 1. 11002-Cádiz, España. 
probado la eficacia del ión $\mathrm{Ce}^{3+}$ como inhibidor catódico para esta aleación ${ }^{[5}$ y 9 -11]. Los resultados obtenidos en estos trabajos se han tomado como punto de partida para el diseño de sistemas de protección basados en el empleo de sales lantánidas ${ }^{[12-15]}$. Así, se ha estudiado el efecto protector frente a la corrosión en $\mathrm{NaCl}$, suministrado por tratamientos de inmersión de la AA5083 en disoluciones de sales de Ce(III) ${ }^{[16-19]}$. De acuerdo con los resultados obtenidos, en estos tratamientos se desarrolla una capa mixta formada por islas de cerio dispersas sobre los intermetálicos catódicos y una película de óxido que cubre la matriz metáli$\mathrm{ca}^{[12]}$. No obstante, para obtener niveles de protección aceptables, se requiere alrededor de $24 \mathrm{~h}$ de tratamiento ${ }^{[12]}$, lo que los hace inviables industrialmente. En este trabajo se propone un método de inmersión acelerado, basado en el aumento de la temperatura del sistema a $323 \mathrm{~K}$, consiguiéndose reducir el tiempo óptimo de tratamiento hasta sólo unos pocos minutos de duración.

\section{MATERIALES Y PROCEDIMIENTO EXPERIMEN- TAL}

Para el desarrollo de este trabajo se emplearon muestras de $30 \times 25 \times 4 \mathrm{~mm}$ de la aleación AA5083. La composición de esta aleación, en tanto por ciento en masa, es la siguiente: $4,9 \% \mathrm{Mg} ; 0,5 \% \mathrm{Mn}$; $0,13 \% \mathrm{Si} ; 0,3 \% \mathrm{Fe} ; 0,03 \% \mathrm{Ti} ; 0,08 \% \mathrm{Cu} ; 0,13 \%$ $\mathrm{Cr}$; resto de Al. Antes de ser tratadas, las muestras se pulieron en papel $\mathrm{SiC}$ hasta un acabado de 500 grits. Posteriormente, fueron desengrasadas con etanol y limpiadas cuidadosamente con agua destilada. Para los tratamientos de inmersión se emplearon disoluciones acuosas aireadas de $\mathrm{Ce}\left(\mathrm{NO}_{3}\right)_{3}$ y $\mathrm{CeCl}_{3}$ 0,005 M, a 323K.

$\mathrm{El}$ aspecto superficial de las capas desarrolladas se estudió mediante Microscopía Electrónica de
Barrido (SEM) en un microscopio JEOL 820-SM. Su composición fue analizada mediante Espectroscopía de Energía Dispersiva (EDS) en Espectrómetro AN-10000 LINK conectado al citado microscopio.

La resistencia frente a la corrosión de las muestras tratadas fue evaluada haciendo uso de la Espectroscopía de Impedancia Electroquímica (EIS), en disolución aireada de $\mathrm{NaCl}$ al 3,5\%, para analizar de forma discriminada la respuesta electroquímica de nuestro sistema. La realización de estas medidas se llevó a cabo en una celda plana K235 de Parc EG\&G, haciendo uso de un potenciostato de Solartron modelo 1287 acoplado a un analizador de respuesta en frecuencia (FRA) de Solartron, modelo SI 1255. Como electrodo de referencia se empleó uno de $\mathrm{Ag} / \mathrm{AgCl}$ de Crison $(0,207$ $\mathrm{mV} / \mathrm{SHE}$ ).

\section{RESULTADOS Y DISCUSIÓN}

El método seguido para el desarrollo de capas de conversión consistió en tratamientos de inmersión en disoluciones de $\mathrm{Ce}\left(\mathrm{NO}_{3}\right)_{3}$ o $\mathrm{CeCl}_{3} 0,005 \mathrm{M}$, a $323 \mathrm{~K}$ durante tiempos comprendidos entre 0 y 60 min. En la figura 1 a) se muestra una imagen SEM correspondiente a una muestra tratada en $\mathrm{Ce}\left(\mathrm{NO}_{3}\right)_{3}$ durante $15 \mathrm{~min}$. Se pueden observar las islas de alto contenido en cerio, (Fig. 1 c). El análisis EDS confirma que el cerio precipita, preferentemente, sobre los intermetálicos de $\mathrm{Al}(\mathrm{Mn}, \mathrm{Fe}, \mathrm{Cr})$, (Fig. 1 d). También, se puede apreciar que en el EDS de la matriz metálica no se encuentra cerio después del tratamiento, (Fig. 1 b). Así, se puede concluir que, mediante estos tratamientos desarrollamos una capa mixta que consiste en islas de cerio situadas sobre los precipitados catódicos de la aleación, y una capa de óxido/hidróxido de aluminio sobre la matriz metálica.
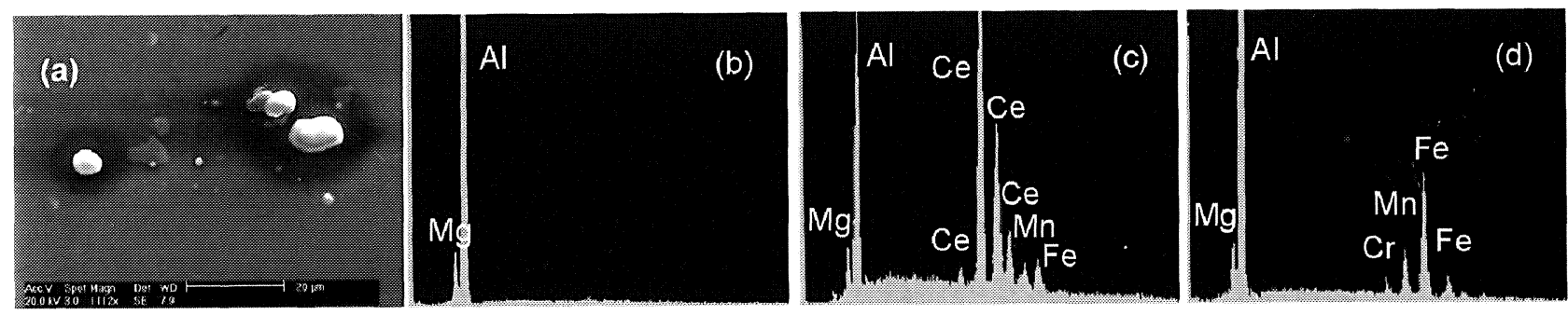

Figura 1. (a) Imagen SEM correspondiente a una muestra tratada durante 15 min en inmersión en $\mathrm{Ce}\left(\mathrm{NO}_{3}\right)_{3}$ a $323 \mathrm{~K}$. EDS adquiridos sobre (b) la matriz metálica, (c) isla de cerio, (d) sustrato de la isla de cerio.

Figure 1. (a) SEM image corresponding to a sample treated during $15 \mathrm{~min}$ by $\mathrm{Fl}$ in $\mathrm{Ce}\left(\mathrm{NO}_{3}\right)_{3}$ at $323 \mathrm{~K}$. EDS acquired on (b) metallic matrix, (c) cerium island and (d) cerium island substrate. 
Con objeto de analizar de forma individualizada la contribución de cada una de las interfases presentes en el sistema se han realizado medidas mediante EIS en disolución de $\mathrm{NaCl}$ al 3,5 \% de las muestras tratadas en cerio. En trabajos previos $^{[12]}$, se ha puesto de manifiesto que la respuesta electroquímica de la aleación AA5083 en disolución de $\mathrm{NaCl}$ puede reproducirse haciendo uso de un circuito equivalente como el de la figura 2 a). En este circuito, $\mathrm{R}_{\mathrm{e}}$ es la resistencia del electrolito; $\mathrm{R}_{\text {int }}-\mathrm{C}_{\text {int }}$ es la malla asociada con las reacciones que tienen lugar en el entorno de los intermetálicos; $\mathrm{R}_{\mathrm{ca}}-\mathrm{C}_{\mathrm{ca}}$ representa la respuesta de la capa; $\mathrm{R}_{\mathrm{T}}$ es la resistencia de transferencia de carga; y $\mathrm{C}_{\mathrm{dl}}$ la capacidad de la doble capa.

Por otro lado, de acuerdo con trabajos publica$\mathrm{dos}^{[12]}$, en presencia de cerio, el proceso de corrosión alcalina localizada queda frenado. De esta forma, la respuesta correspondiente a los intermetálicos se minimiza, desapareciendo la malla $\mathrm{R}_{\text {int }}-\mathrm{C}_{\text {int }}$. En este caso, la respuesta electroquímica del sistema se modela mediante un circuito como el de la figura 2 b). De acuerdo con las consideraciones efectuadas anteriormente, este circuito puede emplearse para simular la respuesta electroquímica en disolución de $\mathrm{NaCl}$ de las muestras tratadas por inmersión en la disolución de sal de cerio. En dicho circuito, la malla $\mathrm{R}_{\mathrm{C}}-\mathrm{C}_{\mathrm{C}}$ representaría la respuesta de la capa mixta desarrollada sobre la aleación durante el tratamiento. Según el trabajo referido ${ }^{[12]}$ esta respuesta se pone de manifiesto en las zonas del espectro correspondiente a las más altas frecuencias. Por esta razón, para llevar a cabo la caracterización electroquímica de la capa mixta sólo es necesario analizar la zona del espectro comprendida entre $10 \mathrm{kHz}$ y $1 \mathrm{~Hz}^{[12]}$.

Los espectros registrados de todas las muestras tratadas presentaron una apariencia similar. En la figura 3 se muestran ejemplos de espectros de impedancia electroquímica adquirido para las muestras tratadas en $\mathrm{Ce}\left(\mathrm{NO}_{3}\right)_{3}$ y $\mathrm{CeCl}_{3}$. Como referencia, se ha incluido el espectro de impedancia de la muestra sin tratar. El arco que aparece en el diagrama de Nyquist para la muestra sin tratar corresponde a la superposición de la respuesta debida a los intermetálicos y a la capa de alúmina ${ }^{[12]}$. Para las muestras tratadas, este arco representa la respuesta de la capa mixta desarrollada ${ }^{[12]}$. Así, en la figura 2 se puede apreciar cómo este arco es de mayor diámetro comparado con la muestra sin tratar. Esto implica un mayor nivel de protección, lo cual es evidente en los altos valores de $|Z|$ en el diagrama de Bode de la figura 3. Esta protección
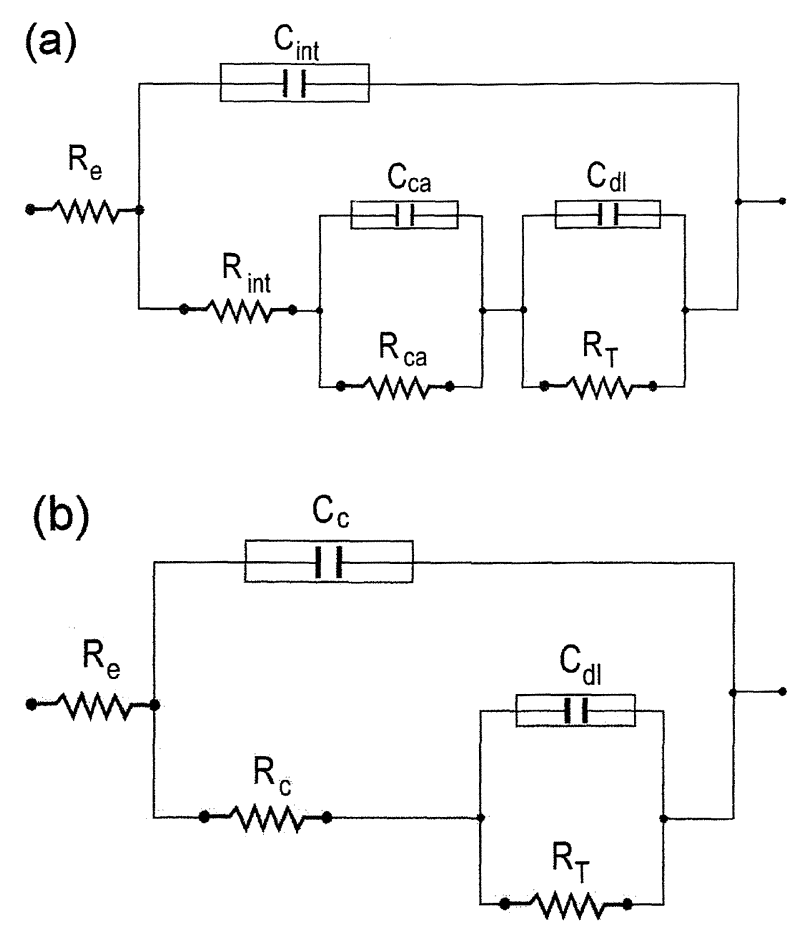

Figura 2. Circuitos equivalentes propuestos para simular la respuesta electroquímica en $\mathrm{NaCl}$ para muestras (a) sin tratar y (b) muestras tratadas.

Figure 2. (Electrical models used to simulate the electrochemical response in $\mathrm{NaCl}$ solutions of (a) untreated and (b) treated samples.

viene dada por el bloqueo de las zonas catódicas. Por otro lado, este mayor nivel de protección también se puede apreciar el diagrama de Bode para $\theta$-f. Así, se puede apreciar que para las muestras tratadas, el $\theta_{\max }$ se mantiené para un mayor rango de frecuencias.

Las conclusiones manifestadas en el párrafo anterior se ven reflejadas en los valores de los elementos que forman el circuito equivalente del sistema. En la tabla I se exponen los valores obtenidos para cada elemento del circuito mostrado en la figura $2 \mathrm{~b}$ ) después de simular los datos experimentales. Se puede apreciar que los valores óptimos se obtienen para tratamientos de $15 \mathrm{~min}$ de duración en disoluciones, tanto de $\mathrm{Ce}\left(\mathrm{NO}_{3}\right)_{3}$ como de $\mathrm{CeCl}_{3}$ a $323 \mathrm{~K}$.

Comparando los valores de $\mathrm{C}_{\mathrm{c}}$ correspondientes a las muestras tratadas, se puede observar que hay una disminución de este parámetro para las muestras tratadas hasta alcanzar su mínimo para 15 min de tratamiento, para luego volver a aumentar. Comparando dicho mínimo con el valor de $\mathrm{C}_{\mathrm{c}}$ para la muestra sin tratar, aquel es menor que el segundo. Asumiendo la siguiente expresión $C=\varepsilon_{0} \cdot \varepsilon \cdot S / d$, queda indicado que el espesor de la película mixta 
Estudio mediante EIS de capas lantánidas de conversión desarrolladas mediante activación térmica sobre aleaciones de $\mathrm{Al}-\mathrm{Mg}$ M. Bethencourt, F.J. Botana, M.J. Cano, M. Montero y M. Marcos
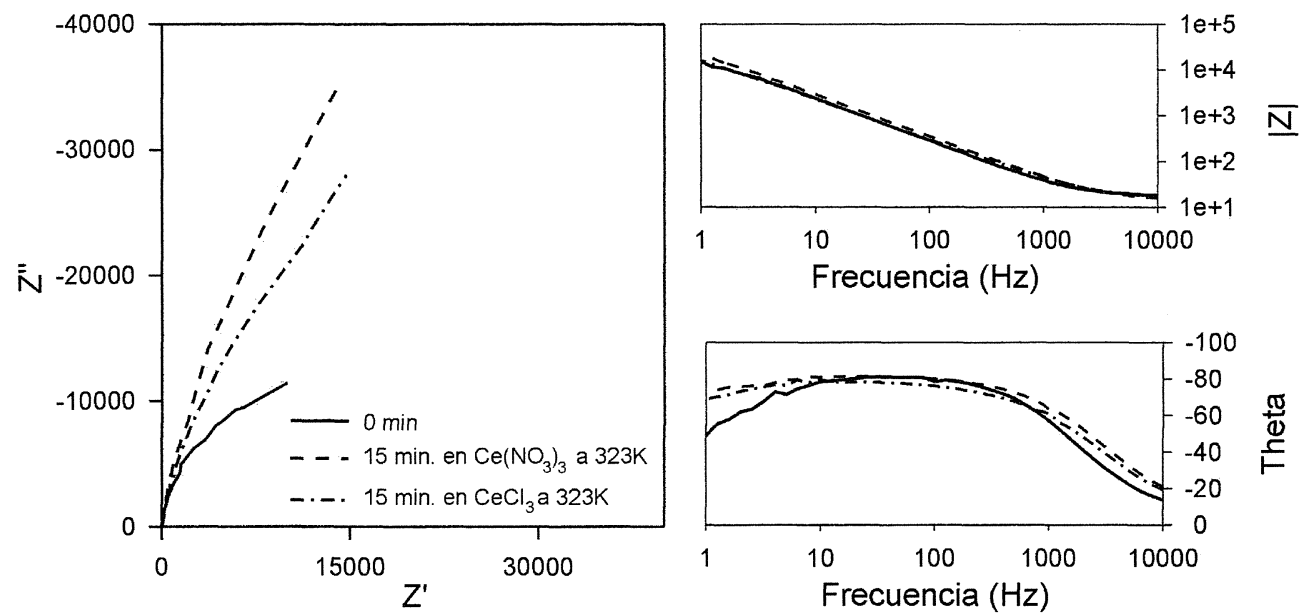

Figura 3. Espectros $\mathrm{EIS}$ obtenidos en $\mathrm{NaCl}$ para muestras tratadas en $\mathrm{Ce}\left(\mathrm{NO}_{3}\right)_{3}$ y $\mathrm{CeCl}_{3}$.

Figure 3. EIS spectra obtained in $\mathrm{NaCl}$ for treated samples in $\mathrm{Ce}\left(\mathrm{NO}_{3}\right)_{3}$ and $\mathrm{CeCl}_{3}$.

Tabla I. Valores de R y C obtenidos del ajuste de los datos experimentales

Table I. Values of $R$ and $C$ obtained by fitting the experimental data

\begin{tabular}{cccccc}
\hline Disolución t. (min) & $\begin{array}{c}\mathbf{R}_{\mathbf{e}} \\
\left(\Omega . \mathrm{cm}^{2}\right)\end{array}$ & $\begin{array}{c}\mathrm{C}_{\mathbf{c}} \\
\left(\mu \mathrm{F} . \mathrm{cm}^{-2}\right)\end{array}$ & $\begin{array}{c}\mathbf{R}_{\mathbf{c}} \\
\left(\mathbf{k} \Omega . \mathrm{cm}^{2}\right)\end{array}$ & $\Delta \mathbf{R}_{\mathrm{ca}}$ \\
\hline- & 0 & 17.22 & 8.99 & $22.973\left(\mathrm{R}_{\mathrm{ca}}\right)$ & 1 \\
& 5 & 15.55 & 9.198 & 98.421 & 4.28 \\
& 10 & 14.94 & 8.571 & 104.53 & 4.55 \\
$\mathrm{Ce}\left(\mathrm{NO}_{3}\right)_{3}$ & 15 & 14.117 & .905 & 135.94 & 5.92 \\
& 30 & 14.33 & 10.349 & 116.77 & 5.08 \\
& 60 & 14.26 & 12.072 & 102.07 & 4.44 \\
& 5 & 14.47 & 10.425 & 61.392 & 2.67 \\
& 10 & 13.63 & 11.776 & 89.967 & 3.90 \\
$\mathrm{CeCl}_{3}$ & 15 & 14.95 & 11.563 & 95.516 & 4.16 \\
& 30 & 15.42 & 13.434 & 81.755 & 3.56 \\
& 60 & 14.61 & 12.663 & 80.924 & 3.52 \\
\hline
\end{tabular}

formada en la muestra tratada durante $15 \mathrm{~min}$ es mayor que el correspondiente a la película formada sobre la superficie de la matriz en las muestras sin tratar. Además, también hay que tener en cuenta que, para la muestra sin tratar, el término de $\mathrm{C}_{\mathrm{c}}$ es un promedio, que se ve aumentado por la presencia de los intermetálicos.

El grado de protección dado por la capa mixta también puede ser evaluado en términos de resistencia. Para esta comparación, se han empleado los valores de $\mathrm{R}_{\mathrm{c}}$ correspondientes a las muestras tratadas con el valor de $\mathrm{R}_{\text {ca }}$, correspondiente a la muestra sin tratar. En la tabla I se incluyen los va- lores de aumento de resistencia de la capa, calculados mediante la expresión $\Delta \mathrm{R}_{\mathrm{ca}}=\mathrm{R}_{\mathrm{c}} / \mathrm{R}_{\mathrm{ca}}$. Tal y como se puede apreciar en la tabla, los mejores resultados se obtienen para capas desarrolladas durante 15 min en disoluciones de $\mathrm{Ce}\left(\mathrm{NO}_{3}\right)_{3}$ y $\mathrm{CeCl}_{3}$ a $323 \mathrm{~K}$, con una mejora de la resistencia de 6 y 4 respectivamente, que son de mismo orden de magnitud que las obtenidas para tiempos de tratamiento de $24 \mathrm{~h}$ a temperatura ambiente ${ }^{[12]}$.

\section{CONCLUSIONES}

Los tratamientos por inmersión en disoluciones de Ce(III) de muestras de la aleación AA5083 conducen al desarrollo de una capa mixta, formada por islas de cerio ubicadas sobre los compuestos intermetálicos de carácter catódico, y una película de alúmina que cubre la matriz metálica. Cuando estos tratamientos se efectúan a temperatura ambiente, el tiempo óptimo de tratamiento se ve condicionado por la lentitud con la que se desarrolla la película de óxido sobre la matriz.

La activación térmica de la disolución de Ce(III) provoca un crecimiento más rápido de esta película proporcionando un nivel de protección del mismo orden de magnitud a los tratamientos efectuados a temperatura ambiente, reduciendo el tiempo de tratamiento, aproximadamente, 100 veces. Cuando los tratamientos se desarrollan a $323 \mathrm{~K}$ durante $15 \mathrm{~min}$ en disolución de $\mathrm{Ce}\left(\mathrm{NO}_{3}\right)_{3}$ se consigue un descenso en la actividad del sistema en un factor próximo a 600 , del mismo orden de magnitud que el obtenido para un día de tratamiento a temperatura ambiente. 


\section{Agradecimientos}

Este trabajo ha sido financiado por la Comisión Interministerial de Ciencia y Tecnología, proyecto MAT2001-3477, y por la Junta de Andalucía.

\section{REFERENCIAS}

[1] A. Aballe, M. Bethencourt, F.J. Botana, M.J. Cano y M. MARcos, Corros. Sci. 43 (2001) 1.657-1.674.

[2] A. Aballe, M. Bethencourt, F.J. Botana, M.J. Cano y M. MARCOS, Materials and Corrosion 52 (2001) 344-350.

[3] A. Aballe, M. Bethencourt, F.J. Botana, M. Marcos, J. Pérez y M.A. RodríGuez-Chacón, Mater. Sci. Forum 289-292 (1998) 557-566.

[4] A. Aballe, M. Bethencourt, F.J. Botana, J. Pérez, M. Marcos y M.A. Rodríguez, Rev. Metal. Madrid 34 (1998) 37-41.

[5] A. Aballe, M. Bethencourt, F.J. Botana, J. Pérez, M. Marcos y M.A. Rodríguez, Rev. Metal. Madrid 34 (1998) 47-51.

[6] M.A. Arenas, M. Bethencourt, F.J. Botana, J.J. Damborenea y M. Marcos, Corr. Sci. 43 (2001) 157 170.

[7] A. Aballe, M. Bethencourt, M.J. Cano y M. Marcos, Corrosion Reviews 18(1) (2000) 1-11.
[8] A. Aballe, M. Bethencourt, F.J. Botana, M.J. Cano y M. Marcos, Electrochem. Soc. Proc. 2000-23 (2001) 364-375.

[9] M. Bethencourt, F.J. Botana, M.A. Cauqui, M. Marcos, M.A. Rodríguez y J.M. ROdRÍGUeZ-IZQUiERdo, J. Alloys Comp. 250 (1997) 455-460.

[10] A. Aballe, M. Bethencourt, F.J. Botana y M. Marcos, J. All. Comp. 323-324 (2001) 855-858.

[11] A. Aballe, M. Bethencourt, F.J. Botana, M. Marcos, J. Pérez y M.A. Rodríguez, Rev. Metal. Madrid 33 (1997) 363-369.

[12] A. Aballe, M. Bethencourt, F.J. Botana, M.J. Cano y M. MARCos, Mater. Corros. 53 (2002) 176-184.

[13] A. Aballe, M. Bethencourt, F.J. Botana, M. Marcos y J.M. Sanchez-Amaya, Rev. Metal. Madrid 37 (2001) 49. 62.

[14] F. Serrano y J.J. Damborenea, Rev. Metal. Madrid 34 (1998) 127-131.

[15] M.A. Arenas, R. Rodríguez y J.J. Damborenea, Rev. Metal. Madrid 37 (2001) 591-596.

[16] A.J. Aldykewicz, H.S. IsaAcs y A.J. Davenport, J. Electrochem. Soc. 143 (1996) 147-155.

[17] Y.C. Lu y M.B. Ives, Corros. Sci. 37 (1995) 145-153.

[18] M. Dabala, L. Armelao, A. Buchberger y I. Calliary, Applied Surface Science 172 (2001) 312-320.

[19] K. Aramaki, Corros. Sci. 43 (2001) 2.201-2.209. 